This is an electronic reprint of the original article. This reprint may differ from the original in pagination and typographic detail.

\author{
Author(s): Palosaari, Mikko; Käyhkö, Marko; Kinnunen, Kimmo; Laitinen, Mikko; Julin, Jaakko; \\ Malm, Jari; Sajavaara, Timo; Doriese, W. B.; Fowler, J.; Reintsema, C.; Swetz, D.; \\ Schmidt, D.; Ullom, J. N.; Maasilta, Ilari
}

Title: $\quad$ Broadband Ultrahigh-Resolution Spectroscopy of Particle-Induced X Rays : Extending the Limits of Nondestructive Analysis

Year: $\quad 2016$

Version:

Please cite the original version:

Palosaari, M., Käyhkö, M., Kinnunen, K., Laitinen, M., Julin, J., Malm, J., Sajavaara, T., Doriese, W. B., Fowler, J., Reintsema, C., Swetz, D., Schmidt, D., Ullom, J. N., \& Maasilta, I. (2016). Broadband Ultrahigh-Resolution Spectroscopy of Particle-Induced $X$ Rays : Extending the Limits of Nondestructive Analysis. Physical Review Applied, 6(2), Article 024002. https://doi.org/10.1103/PhysRevApplied.6.024002

All material supplied via JYX is protected by copyright and other intellectual property rights, and duplication or sale of all or part of any of the repository collections is not permitted, except that material may be duplicated by you for your research use or educational purposes in electronic or print form. You must obtain permission for any other use. Electronic or print copies may not be offered, whether for sale or otherwise to anyone who is not an authorised user. 


\title{
Broadband Ultrahigh-Resolution Spectroscopy of Particle-Induced X Rays: Extending the Limits of Nondestructive Analysis
}

\author{
M. R. J. Palosaari, ${ }^{1}$ M. Käyhkö, ${ }^{2}$ K. M. Kinnunen, ${ }^{1}$ M. Laitinen, ${ }^{2}$ J. Julin, ${ }^{2}$ J. Malm, ${ }^{1,2}$ T. Sajavaara, ${ }^{2}$ W. B. Doriese, ${ }^{3}$ \\ J. Fowler, ${ }^{3}$ C. Reintsema, ${ }^{3}$ D. Swetz, ${ }^{3}$ D. Schmidt, ${ }^{3}$ J. N. Ullom, ${ }^{3}$ and I. J. Maasilta, \\ ${ }^{1}$ Nanoscience Center, Department of Physics, University of Jyvaskyla, \\ P.O. Box 35, FI-40014 Jyvaskyla, Finland \\ ${ }^{2}$ Accelerator Laboratory, Department of Physics, University of Jyvaskyla, \\ P.O. Box 35, FI-40014 Jyvaskyla, Finland \\ ${ }^{3}$ National Institute of Standards and Technology, Boulder, Colorado 80305, USA \\ (Received 25 August 2015; revised manuscript received 24 March 2016; published 4 August 2016)
}

\begin{abstract}
Nondestructive analysis (NDA) based on x-ray emission is widely used, for example, in the semiconductor and concrete industries. Here, we demonstrate significant quantitative and qualitative improvements in broadband x-ray NDA by combining particle-induced emission with detection based on superconducting microcalorimeter arrays. We show that the technique offers great promise in the elemental analysis of thin-film and bulk samples, especially in the difficult cases where tens of different elements with nearly overlapping emission lines have to be identified down to trace concentrations. We demonstrate the efficiency and resolving capabilities by spectroscopy of several complex multielement samples in the energy range 1-10 keV, some of which have a trace amount of impurities not detectable with standard silicon drift detectors. The ability to distinguish the chemical environment of an element is also demonstrated by measuring the intensity differences and chemical shifts of the characteristics $x$-ray peaks of titanium compounds. In particular, we report measurements of the $K \alpha / K \beta$ intensity ratio of thin films of TiN and measurements of Ti $K \alpha$ satellite peak intensities in various Ti thin-film compounds. We also assess the detection limits of the technique, comment on detection limits possible in the future, and discuss possible applications.
\end{abstract}

DOI: 10.1103/PhysRevApplied.6.024002

\section{INTRODUCTION}

$\mathrm{X}$-ray emission spectroscopy is a ubiquitous technique to study the elemental composition of materials. Different versions of the technique exist, varying either in the type of excitation used to generate the characteristic $\mathrm{x}$ rays or in the method of detecting them. Typically, high-energy electrons, ions, or $\mathrm{x}$ rays are used for excitation, with detectors operating in either the energy-dispersive (EDS) or the wavelength-dispersive (WDS) mode. The choice of the excitation source depends on many factors, with electronbeam and $\mathrm{x}$-ray sources being most common. Here, we focus on ion-beam excitation, or the so-called particleinduced x-ray emission (PIXE) technique, most often performed with $2-3-\mathrm{MeV}$ protons, because they give the best x-ray yield for elements between $Z=20$ and 40 [1].

There are several reasons to use particle excitation. Compared to electron-beam excitation, there are at least the following advantages: (i) The accelerated particle beam in PIXE can be taken out of the vacuum to study large or delicate samples (artifacts, biological samples, etc.), (ii) particle beams penetrate much deeper into the sample and can be used to study the subsurface composition, whereas

*maasilta@jyu.fi electron beams give information only from the surface, and, most importantly, (iii) due to the orders-of-magnitudeheavier mass of the proton, the background brehmsstrahlung, which in many cases limits sensitivity, is much reduced. Compared to conventional x-ray-induced fluorescence, PIXE (i) does not suffer from the presence of characteristic peaks of the $\mathrm{x}$-ray tube anode material and (ii) gives more sensitivity for thin samples and elements $Z>20$ due to a lower background in the high-energy range [2].

In any spectroscopic experiment, the ability to detect energy differences as small as possible is always sought after, so that close-lying emission lines in complex samples can be resolved. In addition, in x-ray emission measurements the energy resolution of the detector often sets the limit on the impurity levels that can be measured, as the signal-to-background ratio improves with narrower lines. The most commonly used detector in PIXE measurements is the silicon drift detector (SDD), which is energy dispersive and fairly cheap, offers high count rates, up to $100 \mathrm{kcps}$ without resolution degradation, and has a wide dynamic energy range. However, even the optimal energy resolution of the SDD (typically around $130 \mathrm{eV}$ at $6 \mathrm{keV}$ ) is quite poor and limits the detection of elements with overlapping excitations and low impurity levels. On the 
other hand, wavelength-dispersive detectors have excellent energy resolution (around $1 \mathrm{eV}$ at $6 \mathrm{keV}$ ) [3], but their limited energy range and small solid angle limit their use to experiments where either only a small range in energy is of interest or where high-excitation beam currents and long measurement times are possible.

Here, we apply a detector instrument to PIXE that combines the advantages of the wide energy range, high efficiency, and simplicity of the energy-dispersive detection with ultrahigh-energy resolution and high sensitivity, by using arrays of superconducting transition-edge sensor (TES) x-ray microcalorimeters operated at $0.1 \mathrm{~K}[4,5]$. Although TES sensors have been used before in materials analysis, often their applicability in real-life problems is compromised by their inherently low count-rate capability. However, we obtain competitive counting rates (approximately kcps) and collecting areas by using large arrays with hundreds of individual detector elements. This countrate capability is combined with a full width at half maximum (FWHM) energy resolution demonstrated to be $3 \mathrm{eV}$ at $6 \mathrm{keV}$ in the best cases [6]. Thus, we have a unique instrument for elemental analysis that combines the best aspects of traditional EDS and WDS instruments. Similar TES arrays have recently also been used in tabletop $\mathrm{x}$-ray absorption spectroscopy experiments [7] and in synchrotron-based x-ray spectroscopy [8].

In this study, we report the wide energy range, ultrahighresolution PIXE elemental-analysis results on several kinds of thin-film and bulk samples. In addition to identifying correctly elements from standard samples containing over 60 different trace elements, we also present several results on thin-film $\mathrm{Ti}$ compounds of $\mathrm{Ti}, \mathrm{TiO}_{2}$, and TiN. We measure the chemical shifts of the positions of the Ti $K \alpha$ and $K \beta$ peaks in atomic-layer-deposited $\mathrm{TiO}_{2}$ while collecting data in the full energy range $1-10 \mathrm{keV}$, determine the changes in the $K \alpha / K \beta$ intensity ratios among the different films with high precision, and observe multipleionization satellite emission of the Ti $K \alpha$ transition. We also study ppm-level impurity contents of several common $\mathrm{Co}-$ and $\mathrm{Pb}$-containing pigment materials, discovering low-level impurities, unresolvable with the standard SDD detector, that may be used to fingerprint the material source. A discussion on possible future detection limits and applications is also presented.

\section{SUPERCONDUCTING DETECTOR TECHNOLOGY}

The maturation of low-temperature detector technology enables their use in many experiments ranging in frequency from terahertz bolometry [9] to $\gamma$-ray detection [10]. The detector type used here, a TES microcalorimeter, is an energy-dispersive detector that is based on the sharp resistive transition between the normal state and the superconducting state of a thin film [11]. When a photon hits an absorber material that is coupled to the superconducting thin film, the temperature of the film first quickly rises and then falls back to the steady state with a time constant $\tau$ set by the heat capacity $C$ and thermal conductance $G$ and the reduction in Joule heating associated with the increase in resistance, causing a pulse-shaped change in the resistance of the device. This resistance change causes a pulse in the current, which is read out with a superconducting quantum interference device (SQUID) [11]. SQUIDs are well suited for reading out TESs thanks to their low noise, low power dissipation, and low input impedance $[12,13]$. TES devices optimized for x-ray detection are relatively slow (effective time constants around $0.1-1 \mathrm{~ms}$ ), which limits their count rate to $100-1000 \mathrm{cps}$, but they have high-energy resolution when operated at temperatures around $0.1 \mathrm{~K}$ (in the best cases, below $2 \mathrm{eV}$ at $6 \mathrm{keV}$ [14]). However, with an array of hundreds of TES detectors, the total count rate can be beyond kcps [10]. Another advantage of the array format is that the active detector area, and thus the collection efficiency, is increased to keep the irradiation times shorter, so that the possible damage to delicate samples in a PIXE measurement can be limited. The major motivation for using TES detectors is their exceptionally good energy resolution for energy-dispersive detectors. In addition, within the designed operational energy range of a particular TES detector, the energy resolution is not degraded as a function of energy [5], in contrast to SDD detectors, where it scales as $\sqrt{E}$.

The energy-resolution limit is typically dominated by the unavoidable thermodynamic fluctuations of energy between the sensor and the heat bath, leading to an expression for the theoretical FWHM energy resolution $\Delta E=5.6 \sqrt{k_{B} T^{2} C / \alpha}$, where $k_{B}$ is the Boltzmann constant, $T$ is the temperature of the sensor, $C$ is its heat capacity, and $\alpha=(T / R) \partial R / \partial T$ is the dimensionless parameter describing the steepness of the superconducting transition [5]. The above result is valid for sensors with $G \sim T^{3}$, in the optimal case of $\alpha \gg 1$, without excess noise sources and in the limit $T \gg T_{\text {bath }}$ and $\beta \ll 1$, where $T_{\text {bath }}$ is the temperature of the refrigerating stage (the sensor temperature is always higher due to Joule heating), and $\beta=(I / R) \partial R / \partial I$ with $I$ the current. In addition, optimal filtering and a constant pulse shape are assumed. We see that lowering the operational temperature is the best way to reduce $\Delta E$. The practical limits of refrigeration technology (adiabatic demagnetization and He dilution refrigeration) often set the lower limit to around $T \sim 0.1 \mathrm{~K}$. It then seems that, by decreasing $C$ and increasing $\alpha$, even lower values could be obtained. However, these parameters are hard to change, because the ratio $C / \alpha$ also sets the upper limit in energy where the sensors can be operated without saturation [15]. Thus, for $\mathrm{x}$-ray calorimetry up to the $10-\mathrm{keV}$ range, any significant advances of resolution below $1-2 \mathrm{eV}$ are not straightforward with TES sensors.

Pioneering work on using single-pixel x-ray TES microcalorimeters in electron-beam-induced $\mathrm{x}$-ray spectroscopy 
of materials was performed at NIST Boulder at the turn of the millennium [16,17], with the result that an electron microprobe analysis with a small array of TES sensors is now commercially available, with an energy resolution below $10 \mathrm{eV}$ at $1.7 \mathrm{keV}$ [18]. In Ref. [17], it is reported that the single-pixel TES-based electron microprobe spectrometer operating up to $10 \mathrm{keV}$ has a FWHM resolution of $4.5 \mathrm{eV}$ at $5.9 \mathrm{keV}$, with high efficiency and a maximum count rate of approximately $1000 \mathrm{cps}$. In addition, a singlepixel TES sensor is tested with PIXE, as well [19], but only with a far-from-optimal energy resolution around $18 \mathrm{eV}$ at $1.7 \mathrm{keV}$ and a count rate of approximately 50 cps. Our instrument achieves almost an order-ofmagnitude improvement in energy resolution, and over an order of magnitude in count rate, making it a truly practical PIXE spectrometer.

\section{EXPERIMENTS}

\section{A. Detector setup}

The 160-pixel TES array and the time division multiplexed SQUID readout [13] used in the experiments are fabricated at NIST Boulder. A single TES pixel consists of an about 300-nm-thick superconducting $\mathrm{Mo} / \mathrm{Cu}$ bilayer with a critical temperature $T_{C}$ around $100 \mathrm{mK}$. The 500nm-thick normal-metal $\mathrm{Cu}$ strips are deposited on top of the bilayer to suppress local $T_{C}$ variation at the edges [20] and to suppress noise [21]. The absorber, coupled directly to the superconducting film, is a $350 \times 350 \times 2.5 \mu \mathrm{m}^{3}$ bismuth block. $\mathrm{Bi}$ is used because of its advantageous combination of high x-ray absorption and low heat capacity. A collimator chip with a $320 \mu \mathrm{m} \times 305 \mu \mathrm{m}$ aperture size for each pixel sits on top of the array chip to prevent $x$-ray hits outside the absorber area.

The experiments presented in this paper are performed with a total of 30-60 pixels, a $65-\mathrm{mK}$ bath temperature, and a $2.01-\mathrm{MeV}$ proton beam striking the samples in vacuum. A pulse-tube precooled cryogen-free adiabatic demagnetization refrigerator [22] is used to cool the detectors and the readout, which are located inside a specially designed extension structure [6] to facilitate close proximity to the samples (Fig. 1). The sample and the detector vacuums are separated by an AP3.3 ultrathin polymer/Al silicon grid $\mathrm{x}$-ray window from Moxtek Inc., allowing efficient x-ray transmission above $1-\mathrm{keV}$ energies. In addition, to stop backscattered protons from hitting the detector setup, an additional 125- $\mu \mathrm{m}$-thick Be foil is placed in front of the vacuum window. The extension is positioned at a $90^{\circ}$ angle with respect to the proton-beam axis, and the sample surface is tilted $45^{\circ}$ with respect to it. Thin infrared filters fabricated in house (280-nm SiN membrane supporting a $225-\mathrm{nm} \mathrm{Al} \mathrm{film)} \mathrm{are} \mathrm{used} \mathrm{at} \mathrm{the} \mathrm{three} \mathrm{shielding} \mathrm{stages}$ $(60 \mathrm{~K}, 3 \mathrm{~K}$, and $50 \mathrm{mK}$ ) of the extension, to reduce unwanted power loading on the detectors. Further details of the measurement geometry and the setup can be found in

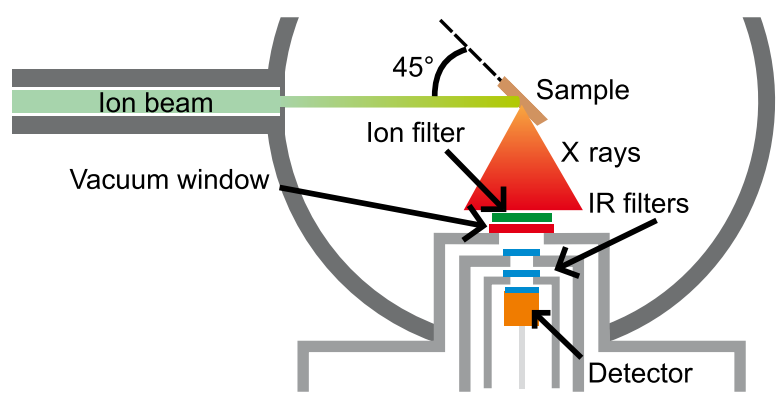

FIG. 1. A schematic of the detector setup. The sample and the detector vacuums are separated by an X-ray vacuum window, with an additional $\mathrm{Be}$ foil as an ion filter in front of the vacuum window. Thin SiN/Al infrared filters are used at the three shielding stages $(60 \mathrm{~K}, 3 \mathrm{~K}$, and $50 \mathrm{mK})$ of the extension.

Ref. [6], where the FWHM energy resolution $3.06 \mathrm{eV}$ of the best pixel in our setup is also reported. In contrast to Ref. [6], however, the distance between the detector and the samples is reduced from 30 to $15 \mathrm{~cm}$, increasing the collection efficiency by a factor of 4 . An Amptek $\mathrm{X}$-123SDD silicon drift detector is used as a reference detector, with measurements performed in air.

\section{B. Reference samples and efficiency calibration of the TES array}

To demonstrate the capabilities of TES PIXE, two NIST standard reference material (SRM) samples with known compositions, a SRM 1157 stainless-steel sample (Fig. 2) and a SRM 611 glass sample (Fig. 3), are measured both with the TES-PIXE setup and with the SDD detector. SRM 1157 has 12 minor elements in addition to $\mathrm{Fe}: \mathrm{C}, \mathrm{Si}, \mathrm{P}, \mathrm{S}$, $\mathrm{Mo}, \mathrm{V}, \mathrm{Cr}, \mathrm{Mn}, \mathrm{Ni}, \mathrm{Cu}, \mathrm{W}$, and $\mathrm{Co}$, with concentrations ranging from $40 \mathrm{ppm}$ to $6 \%$, whereas SRM 611 has altogether 61 trace elements in a glass support matrix with concentrations ranging from 100 to $500 \mathrm{ppm}$. The glass matrix has a nominal composition of $72 \% \mathrm{SiO}_{2}, 14 \%$ $\mathrm{Na}_{2} \mathrm{O}, 12 \% \mathrm{CaO}$, and $2 \% \mathrm{Al}_{2} \mathrm{O}_{3}$. The average count rate for a single TES pixel is $9.3 \mathrm{cps}$, leading to a total count rate of about $550 \mathrm{cps}$ for 60 pixels. The SDD count rate is $1400 \mathrm{cps}$. The measurement times ( $4 \mathrm{~h}$ for TES and $1.13 \mathrm{~h}$ for SDD) are chosen such that the total number of counts is approximately the same. For the TES detector, the conversion from pulse height to energy is achieved by a spline interpolation of the known peak energies, for each pixel separately due to the intrinsic nonlinearity of the TES detector. In the full energy range of $1-10 \mathrm{keV}$, the typical observed pulse height nonlinearity is $(25 \pm 0.3) \%$ at $10 \mathrm{keV}$ compared to a line fitted at the lower energy range. The error gives the variation from cooldown to cooldown. Before a measurement of an unknown sample, the energy calibration is always checked with a dedicated calibration sample, consisting of a thin-film multilayer of $\mathrm{Ge}, \mathrm{Cu}, \mathrm{Cr}$, and $\mathrm{Ti}$ on a Si substrate. The $K \alpha$ and $K \beta$ lines of those elements cover the energy range $1-11 \mathrm{keV}$. The drift 


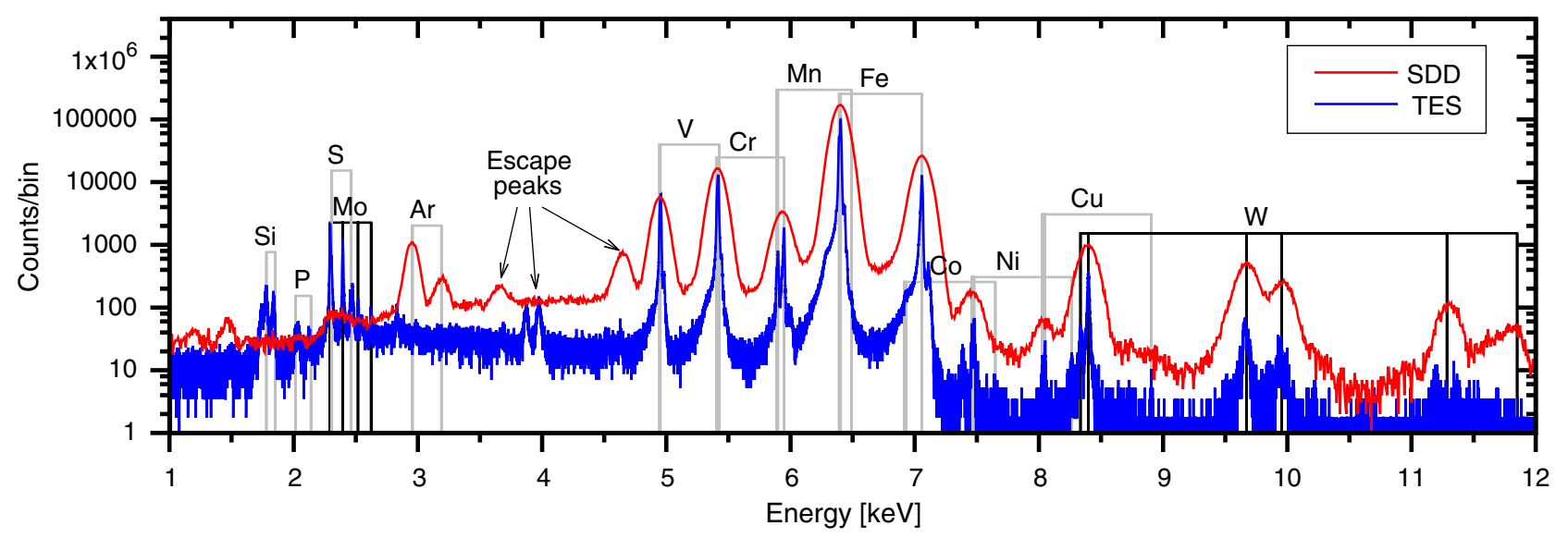

FIG. 2. SRM 1157 reference sample measured with both TES (blue line) and SDD (red line) detectors. The bin width for TES is $0.5 \mathrm{eV}$, and for SDD it is $7 \mathrm{eV}$. The Ar excitations from air can be seen in the SDD data around $3 \mathrm{keV}$ as well as the Si escape peak from the $\mathrm{Fe} \mathrm{x}$ rays at $4.6 \mathrm{keV}$ and from the $\mathrm{Cr}$ at $3.7 \mathrm{keV}$. In the TES data, the Bi escape peaks can be seen just below $4 \mathrm{keV}$.

occurring during the measurement is corrected to the first order [23].

We can see from Figs. 2 and 3 the clear difference in the energy resolution between the SDD and the TES setup. Focusing on the steel sample first (Fig. 2), we observe that, with the TES detector, we can identify all elements in the sample except carbon (its emission below $1 \mathrm{keV}$ is cut by filtering in the setup). In particular, low levels of Co $(280 \mathrm{ppm})$ can be seen, even though its $K \alpha$ lines are close to the dominant $\mathrm{Fe} K \beta$ line at $7 \mathrm{keV}$. Moreover, phosphorus and sulphur lines are also observable above $2 \mathrm{keV}$, even though the concentrations are low-110 and $40 \mathrm{ppm}$, respectively. None of those elements (Co, P, and S) could be identified with the SDD detector. For the more complex sample SRM 611, some of the dense peaks in the energy range 4-8 keV overlap in the SDD data, making the identification of most elements impossible or extremely challenging with SDD detectors, particularly the heavier rare-earth elements $(\mathrm{Ce}, \mathrm{Nd}, \mathrm{Sm}, \mathrm{Gd}, \mathrm{Tb}$, and Dy) whose $L \alpha$ lines are mixed in with the $K \alpha$ lines of the lighter transition metals ( $\mathrm{Sc}, \mathrm{Ti}, \mathrm{V}, \mathrm{Cr}, \mathrm{Mn}, \mathrm{Fe}, \mathrm{Co}$, and $\mathrm{Ni}$ ). In contrast, with the TES detectors, we identify most of the elements (38) that emit $x$ rays in the energy range shown, without any complex peak-fitting analysis. By looking at the observed good peak-to-background ratios of the SRM 611 trace-element peaks measured with the TES detectors, we estimate that roughly over an order-ofmagnitude-lower level impurities at the 10-50-ppm level could have been detected with the same measurement time. As a final note on the SRM 611 spectrum and to put it into perspective, an electron probe analysis with a commercial liquid-nitrogen-cooled semiconductor detector could not identify any of the trace elements.

For the highest count-rate peaks (e.g., Fe in Fig. 2), there is evidence of asymmetric low-energy tailing, which we know originates from the nonidealities of the $\mathrm{Bi}$ absorber.

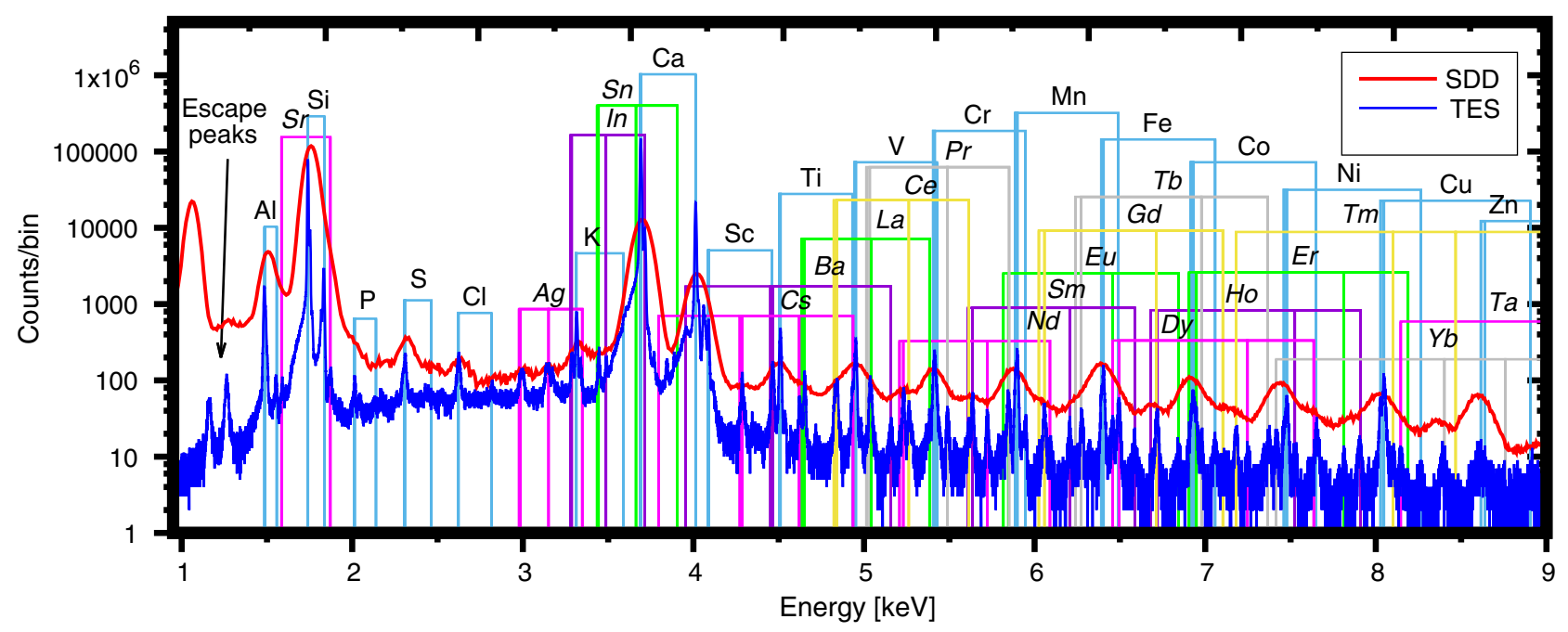

FIG. 3. SRM 611 reference sample measured with both TES (blue line) and SDD (red line) detectors. The bin width for TES is 0.5 eV, and for SDD it is $7 \mathrm{eV}$. The Ar excitations from air can be seen in the SDD data, as well as the Si escape peak from the Ca x rays at $2 \mathrm{keV}$. 
One possibility is that there are long-lived metastable states in Bi that can trap a small part of the heat pulse long enough so that it does not contribute to the measured response. More work is required to clarify this effect.

To be able to discern quantitative information on samples with unknown elemental concentrations, an efficiency calibration of the detector is required. To determine the detector efficiency, the measured data of the SRM samples are analyzed using the GUPIXwin program [24], which calculates the elemental concentrations based on the known physical parameters of the setup. In the GUPIXwin analysis, all the external filters are taken into account, but the to-bedetermined detector setup efficiency is temporarily set to 1 . For the SRM 611 sample, the nominal matrix mass composition $\left(72 \% \mathrm{SiO}_{2}, 14 \% \mathrm{Na}_{2} \mathrm{O}, 12 \% \mathrm{CaO}\right.$, and $2 \%$ $\mathrm{Al}_{2} \mathrm{O}_{3}$ ) is used in the GUPIXwin analysis, whereas for SRM 1157 sample, the matrix composition (consisting mostly of iron) is iterated by GUPIXwin. We do not use the standard fitting procedures of the program; instead, the peak areas are integrated after the continuous background is removed by a visual fit. Then, the concentrations (without the efficiency of the setup) are calculated using the x-ray yields that GUPIXwin calculates. Finally, the detector setup efficiency is calculated as the ratio between the measured concentration without the detector efficiency and the known reference concentration, for all reference energies. In the analysis, statistical uncertainties and uncertainties in the ionization cross sections for $K$ lines (1\%-7\%) [25] and $L$ lines (5\%) [26] are taken into account. The reference values for the concentrations and energies of the analyzed elements are taken from Ref. [27] (preferred average values, Table 8) for SRM 611 and from the NIST certificate for the SRM 1157 sample.

Figure 4 shows the measured efficiency data (dots), compared with the theoretical efficiency curve consisting of

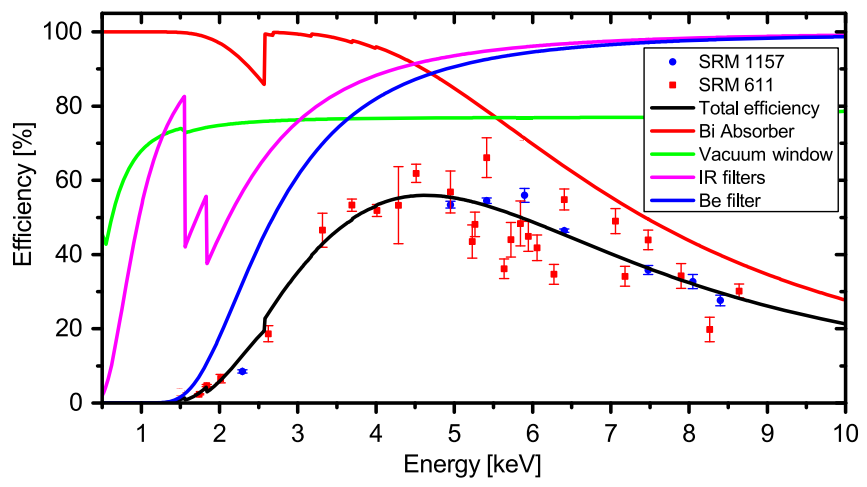

FIG. 4. The measured (squares and circles) and simulated (black line) total efficiency of the TES-PIXE setup. Also plotted are the absorption of the $\mathrm{Bi}$ absorber (red line) and the transmissions of the AP3.3 vacuum window (green line), infrared filters (pink line), and the Be filter (blue line). The efficiency curve is calibrated using the known reference materials NIST SRM 611 (squares) and NIST SRM 1157 (circles). the transmission through the $125-\mu \mathrm{m}$ Be filter used to stop the scattered ions, through the AP3.3 vacuum window, through the 280-nm-thick $\mathrm{Si}_{3} \mathrm{~N}_{4}$ membrane with a 220 -nm $\mathrm{Al}$ coating used as infrared filters at the three stages of the cryostat, and the absorption of the $2.5-\mu \mathrm{m} \mathrm{Bi}$ absorber (using Ref. [28]). We see that the experimental values agree well with the theory and conclude that, within the accuracy of the measurement and the reference data, the calculated theoretical curve can be used to represent the efficiency of the setup. We note that the SRM 1157 points are derived from elements with high concentrations, leading to low uncertainties and very good agreement with the theory. In contrast, some of the SRM 611 points have more scatter. This is natural, as many of the trace-element concentrations of that sample are not so accurately known and are uncertified. In addition, we note that three observed elements $(\mathrm{Al}, \mathrm{Cu}$, and $\mathrm{Mn})$ are excluded from the analysis because of extra fluorescence coming from the sample stage and other parts of the apparatus. It should also be noted that the theoretical efficiency of the vacuum window never reaches a level higher than $77 \%$ below $10-\mathrm{keV}$ energies. This is due to the fact that a thick silicon grid with a $77 \%$ hole area is under the polymer in the AP3.3 vacuum window.

\section{Chemical shifts, intensity ratios, and satellite peaks in Ti-based samples}

Because of the excellent energy resolution of TES detectors, we expect that even chemical effects, where the characteristic $x$ rays of a material are dependent on the chemical environment of a element, can be observed in our setup. The chemical state of an element (oxidation number and chemical bonding) influences its electronic transitions and atomic-energy levels, even those involving the inner shells of the atoms. Different kinds of effects are observed that affect $x$-ray transitions: energy shifts of the characteristic $\mathrm{x}$-ray lines, the alteration of $\mathrm{x}$-ray relative intensity ratios and line shapes, and satellite peak formation [29]. Typically, these kinds of shifts can be resolved only with a wavelength-dispersive and not with an energy-dispersive detector; see, for example, Ref. [30]. With TES detectors in the electron-probe analysis setup, evidence for Fe $L$-shell transition chemical shifts has been seen before [17].

We measure the x-ray spectra of three different titaniumbased thin-film samples-Ti $(400 \mathrm{~nm})$, TiN $(500 \mathrm{~nm})$, and $\mathrm{TiO}_{2}(450 \mathrm{~nm})$ - all on $\mathrm{Si}$ substrates, with TES PIXE, using $2-\mathrm{MeV}$ proton excitation. The TiN and $\mathrm{TiO}_{2}$ are grown with atomic-layer deposition, and the Ti film is electronbeam evaporated. The samples are measured in the order Ti, $\mathrm{TiN}, \mathrm{Ti}, \mathrm{TiO}_{2}$, and $\mathrm{Ti}$, so that any possibility of jumps in the calibration is ruled out by comparing the Ti spectra. Any possible energy drift of a pixel is corrected on the Si $K \alpha$ line, which is common in all of the samples and close enough in energy to Ti so that the error introduced by the gain nonlinearity is insignificant. Each sample is measured 


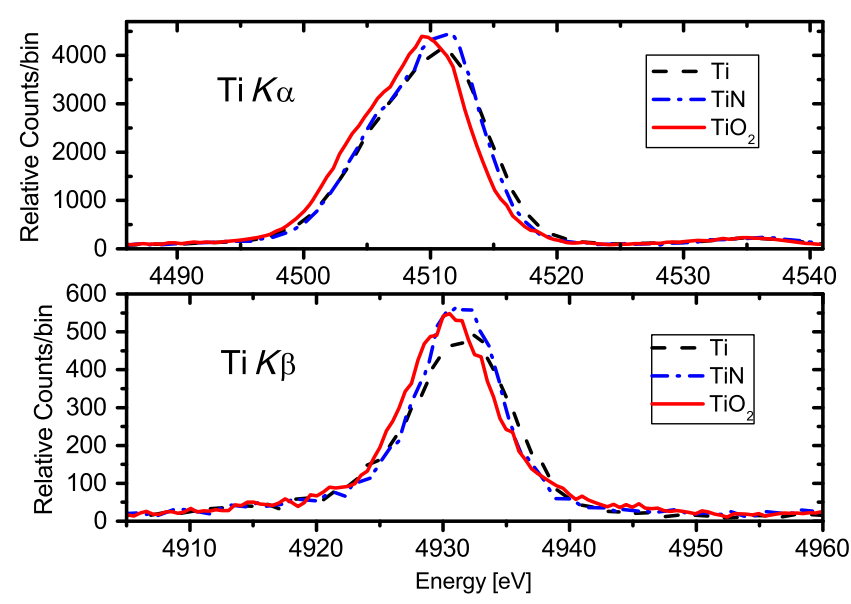

FIG. 5. Ti peaks from the three different $\mathrm{Ti}$ compounds normalized for the $K \alpha$ peak area. The spectra are divided into two plots for clarity. Top: The Ti $K \alpha_{1,2}$, bottom: The Ti $K \beta_{1,3}$. A chemical shift for the $\mathrm{TiO}_{2}$ sample is visible.

for $1 \mathrm{~h}$ with an average count rate of about $8 \mathrm{cps}$ per pixel. The energy calibration of the spectra is done on the Ti sample, using the $K \alpha$ and $K \beta$ peaks of $\mathrm{Si}$ and Ti.

Figure 5 shows the measured Ti $K \alpha$ and $K \beta$ complexes from all three samples. A negative chemical shift of $1.1 \mathrm{eV}$ is seen on the $K \alpha$ complex of the $\mathrm{TiO}_{2}$ sample compared to the pure Ti sample. Also, a slightly smaller shift of $1.0 \mathrm{eV}$ can be seen in the $K \beta$. No measurable shift can be seen in the TiN spectrum. The shift is determined by fitting Gaussians to the peaks and taking the difference between the peak centroids of the $\mathrm{Ti}$ and $\mathrm{TiO}_{2}$ lines. The standard deviation of the peak position for all the lines is $0.3 \mathrm{eV}$ (24 pixels in this measurement), which is well below the observed shift value, giving us confidence on the statistical significance of the result. Moreover, all the shifts have the same negative sign, strong evidence against a random process. In addition, this chemical shift result agrees with $\mathrm{x}$-ray photoelectron spectroscopy measurements [31] and wavelength-dispersive spectrometer measurements [32]. Note that, due to the peak-fitting procedure, chemical shifts much below the FWHM energy resolution of the instrument are observable. The observed $K \beta / K \alpha$ intensity ratios are $(12.5 \pm 0.1) \%$ for $\mathrm{Ti},(13.1 \pm 0.2) \%$ for TiN, and $(13.3 \pm 0.2) \%$ for $\mathrm{TiO}_{2}$. This trend of an increasing $K \beta / K \alpha$ ratio is consistent with previous studies of $\mathrm{Ti}$ and $\mathrm{TiO}_{2}[33-$ $35]$, as the $K \beta / K \alpha$ ratios are shown to depend on the chemical state of $3 \mathrm{~d}$ elements [36]. No literature results for TiN are found. From the above results, we conclude that the accurate measurement of the $K \beta / K \alpha$ intensity ratio is a very useful way to obtain chemical information, as all studied Ti compounds show different values. This is in contrast to the shift in the line position, where only $\mathrm{TiO}_{2}$ shows a measurable effect. Moreover, the measurement of peak ratios is less prone to systematic errors and is not sensitive to energy calibration issues. Note that, in addition to sensitivity to different compounds, one can, in principle, also distinguish between different oxidation states of $\mathrm{Ti}$ in the same compound, i.e., the different oxides $\left(\mathrm{TiO}, \mathrm{TiO}_{2}\right.$, and $\mathrm{Ti}_{2} \mathrm{O}_{3}$ ).

On the high-energy side of the main peaks (e.g., at $4.535 \mathrm{keV}$ ), the spectra show also smaller satellite lines, typical in PIXE measurements. The $K \alpha$ satellite intensity compared to the total $\mathrm{Ti}$ emission is $(4.9 \pm 0.1) \%$ for $\mathrm{Ti}$, $(5.0 \pm 0.1) \%$ for $\mathrm{TiN}$, and $(4.8 \pm 0.1) \%$ for $\mathrm{TiO}_{2}$. This satellite peak is most likely generated during the ion bombardment when $1 s$ and $2 p$ vacancies are created simultaneously (multiple ionization). The $2 p$ vacancy has a longer lifetime than the $1 s$ vacancy. When the inner $1 s$ vacancy deexcites in the presence of this spectator hole, there is a change in the electrostatic potential, leading to shifts in the energy levels and thus changing the energy of the emitted photon [37]. Although multiple-ionization Ti $K \alpha$ satellite peaks have been observed before with ionbeam excitation [38,39], we are not aware of any previous measurements on $\mathrm{Ti}$ thin-film samples or comparisons between different chemical compounds of Ti. As seen from the satellite intensity values, we do not observe any significant chemical sensitivity with the proton-beam excitation. However, literature reports suggest that with heavier projectiles chemical information can be obtained in some cases from the satellite intensities $[40,41]$.

\section{Pigment measurements}

Finally, we also present elemental-analysis data from several pigment samples with unknown impurity content. Pigment analysis is useful in the context of art history, as major-element and trace-impurity content in pigments can pinpoint or rule out certain geographical locations and time periods [42]. As a first example, we show spectra from a cobalt blue $\left(\mathrm{CoAl}_{2} \mathrm{O}_{4}\right)$ pigment sample (pellet compressed from powder), measured using both the TES and SDD detectors (Fig. 6) and a 2-MeV proton beam. The spectra

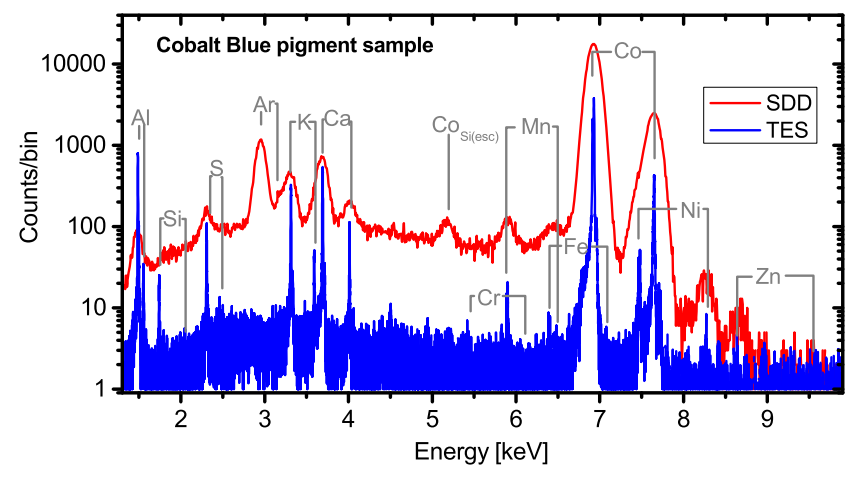

FIG. 6. PIXE spectra of a Co pigment sample using the SDD (red line) and TES (blue line) detectors. The bin width for TES is $0.5 \mathrm{eV}$ and for the SDD $7 \mathrm{eV}$. Cr and Fe trace impurities can be resolved with the TES detectors. 
are collected with a measurement time of 50 (TES) and 10 min (SDD). Many trace impurities can be identified: $\mathrm{S}, \mathrm{K}, \mathrm{Ca}, \mathrm{Cr}, \mathrm{Mn}, \mathrm{Fe}, \mathrm{Ni}$, and $\mathrm{Zn}$. Out of those impurities, $\mathrm{Fe}$ and $\mathrm{Cr}$ are observable only in the TES spectrum and cannot be identified from the SDD spectrum. The estimate for the Fe concentration is $920 \pm 140 \mathrm{ppm}$ and for the Cr $110 \pm 40 \mathrm{ppm}$, calculated with the efficiency curve obtained previously. The TES spectrum shows also weak $\mathrm{Si}$ lines, but they may originate from secondary fluorescence of the vacuum window and/or IR filters.

Two different lead pigments, red lead $\left(\mathrm{Pb}_{3} \mathrm{O}_{4}\right)$ and lead white $\left[2 \mathrm{PbCO}_{2} \cdot \mathrm{Pb}(\mathrm{OH})_{2}\right]$, are also measured in addition to a reference sample of a bulk piece of industrial-grade metallic lead sheet (Fig. 7). The measurement time is 50 min for all samples with the TES detectors and below 10 min for the SDD. Both $M$ - and $L$-shell $\mathrm{Pb}$ transitions are observable at the low and high end of the spectra, respectively. All three samples have clearly different compositions of trace impurities, again many of them identified only with the help of the TES detectors. Interestingly, all samples have traces of gold in the range 1000-2000 ppm. There are also visible peaks from copper in all samples, but we determine that most of the $\mathrm{Cu}$ contribution comes from the sample stage, so the possible sample contributions are unknown. The $\mathrm{Pb}$ red pigment is otherwise surprisingly clean. On the other hand, both the
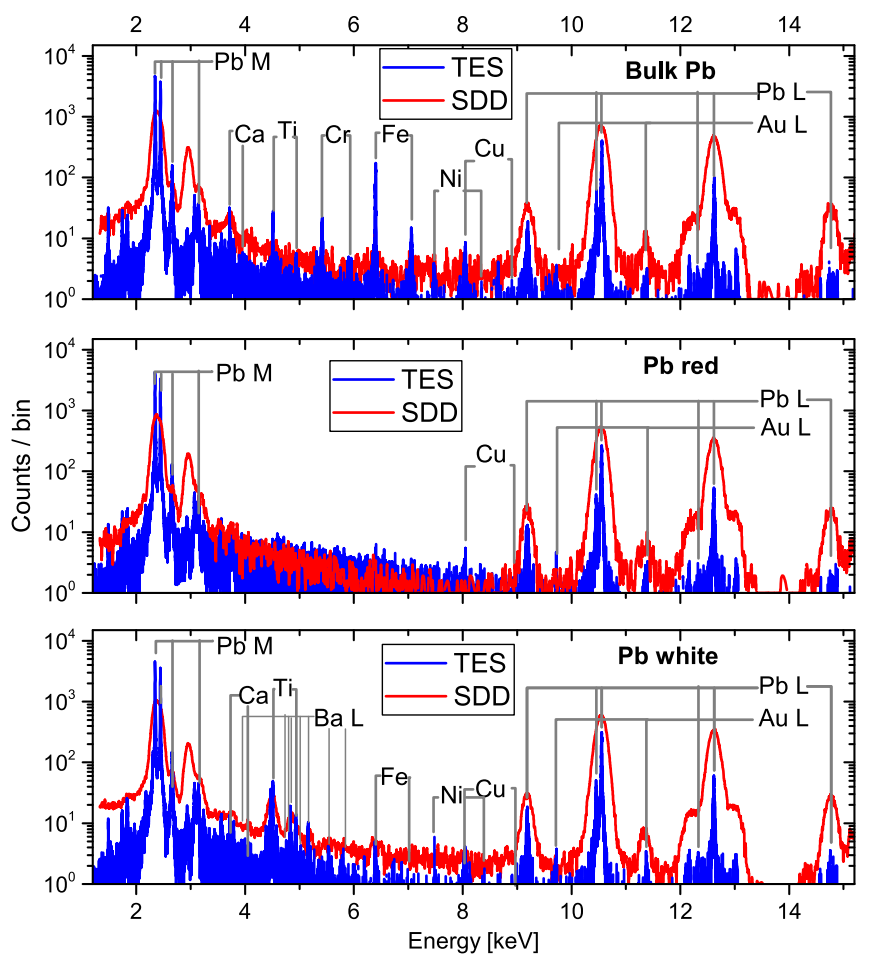

FIG. 7. PIXE spectra of three different $\mathrm{Pb}$ samples: bulk $\mathrm{Pb}$ (top), $\mathrm{Pb}$ red pigment (middle), and $\mathrm{Pb}$ white pigment (bottom), measured with both the SDD (red line) and TES (blue line) detectors. The bin width for TES is $0.5 \mathrm{eV}$ and for the SDD $7 \mathrm{eV}$. bulk $\mathrm{Pb}$ and the $\mathrm{Pb}$ white samples have many additional common impurities: $\mathrm{Ba}, \mathrm{Ca}, \mathrm{Ti}, \mathrm{Fe}$, and $\mathrm{Ni}$, at concentrations $100-9000 \mathrm{ppm}$. The bulk sample has, in addition, $\mathrm{Cr}$ at the 1600-ppm level. Further studies will reveal if all $\mathrm{Pb}$ white samples contain these impurities or not, and whether some of them could serve as a fingerprint. Barium seems especially interesting, as the $\mathrm{Pb}$ red sample does not contain any within the detection limits, whereas the $\mathrm{Pb}$ white contains it at the 8500-ppm level, but the bulk lead only at the 800-ppm level.

\section{E. Analysis of detection limits}

In all the presented spectra, it is clear that PIXE, in general, is well suited for trace-element analysis due to its low background level and that TES PIXE has more sensitivity than standard PIXE using SDD detectors. The detection limits are ultimately determined by Poisson statistics of the random count arrival times. Following Ziebold [17,43], the minimum mass fraction detectability limit at the $95 \%$ confidence level, $C_{\mathrm{MMF}}$, for trace elements and without peak interference can be estimated from

$$
C_{\mathrm{MMF}}=3.29 a / \sqrt{\tau P(P / B)},
$$

where $a$ is an element and matrix-dependent unitless constant of the order of 1 [43], $\tau$ is the measurement time, $P$ is the count rate of the peak in question, and $P / B$ is the peak-to-background ratio in measurement time $\tau$ in a pure sample. Looking at this formula, it is clear that, for a constant measurement time, to improve detection limits one can increase either the peak count rate or the peak-tobackground ratio. Our TES-PIXE setup is extremely well suited for multiple-trace-element detection, because it combines a reasonably good count rate (due to the detector array and high efficiency) with an excellent peak-tobackground ratio due to the high-energy resolution, in a wide energy range. WDS spectrometers can be competitive for single-trace-element analysis but simply cannot operate in a 10-keV energy window in the multiple-trace-element mode. The WDS spectrometer also has a higher theoretical upper limit for the count rate, but, because of the lack of detection efficiency, much higher proton-beam currents must be used to achieve similar count rates, which can be a problem for delicate samples.

Using one of the measured lines, we can also try to estimate the current and future TES-PIXE detection limits using Eq. (1). Using the Co $K \alpha$ peak from the Co pigment spectrum, we estimate $P / B \sim 65500, P \sim 44 \mathrm{cps}$, and thus, for the used measurement time of $50 \mathrm{~min}$, we get $C_{\mathrm{MMF}} \sim 35 \mathrm{ppm}$. The measurement is performed with 31 pixels, so in the future, by upgrading to the full array capability (160 pixels), the estimated detection limit will decrease by a factor of $\sqrt{31 / 160}$ to $15 \mathrm{ppm}$. A further improvement can still be obtained by increasing the proton current and irradiation time, so, for nondelicate samples, 
limits below $1 \mathrm{ppm}$ can be achieved. In addition, the origin of the background is presently under study, and it may be reduced in the future, which would lead to a further reduction in the detection limits. These estimates indicate that TES PIXE has the capability of becoming the most sensitive nondestructive analysis technique.

As a final comment on the detection limits, the above analysis concerns only well-separated peaks. If there is peak overlap, detection limits improve superlinearly with the improving energy resolution. Statham [44] introduces several figures of merit proportional to the analysis speed for cases where overlap is important. For example, he estimates that, if a trace-element peak has to be resolved with overlap from another peak, the analysis speed scales as $P / \mathrm{FWHM}^{3}$, where FWHM is the full-width-at-halfmaximum energy resolution. Thus, TES PIXE can give a factor of $(3 \mathrm{eV} / 130 \mathrm{eV})^{3} \sim 10^{-5}$ smaller detection limits compared to standard SDD detectors in those kinds of cases.

\section{CONCLUSIONS}

We recently developed a powerful x-ray spectroscopic materials analysis tool [6], which utilizes an array of superconducting transition-edge sensors operated at $0.1 \mathrm{~K}$ as the detectors, coupled with an ion-beam (proton) excitation by an accelerator (TES PIXE). Here, we demonstrate the spectroscopic capabilities of the instrument by measuring several complex samples with many elements. The TES detectors clearly give a large advantage over the traditional silicon drift detectors in terms of the capability to identify and resolve a large number (approximately 50) of closely spaced characteristic x-ray peaks over a large energy window (1-10 keV). Much smaller concentrations of trace impurities can be resolved, and in some cases chemical effects are observable (here, in the Ti $K$-shell transitions). Although high-energy resolution can be obtained with wavelength-dispersive methods, as well, they cannot be operated as efficiently and easily over a broad energy range as what we demonstrate here. The superior detection efficiency of TES detectors also means that smaller excitation currents can be used as compared to wavelength-dispersive detection.

Several physical effects are seen, from both thin-film and bulk samples. Chemical shifts of the positions of the Ti $K \alpha$ and $K \beta$ peaks in atomic-layer-deposited $\mathrm{TiO}_{2}$ thin films are observable, while collecting data in the full energy range $1--10 \mathrm{keV}$. Chemical changes in the $K \alpha / K \beta$ intensity ratios between thin films of $\mathrm{Ti}, \mathrm{TiN}$, and $\mathrm{TiO}_{2}$ are measured. Our data also give a comparison of Ti $K \alpha$ multiple-ionization satellite peak intensities in Ti thin-film compounds. We also study ppm-level impurity contents of several common $\mathrm{Co}-$ and $\mathrm{Pb}$-containing pigment materials, discovering low-level impurities, unresolvable with the standard SDD detector, that may possibly be used to fingerprint the material source.

\section{OUTLOOK}

All the measurements discussed here are performed in vacuum. In PIXE, the excitation beam can be brought to atmosphere, as well, and our cryogenic setup does allow the coupling of $x$ rays from air. This means that there are future prospects of studying specimens that are large or specimens that might not survive the vacuum. Collection efficiency will also be improved by operating the full 160-pixel array. Detector fabrication and readout electronics improvements in the future will also allow for the operation of even larger arrays, possibly up to the kilopixel range, with higher perpixel count rates.

We also discuss some possible future directions. In terms of impurity analysis, TES PIXE using large detector arrays offers an unparalleled combination of efficiency, resolution, and large energy range, to identify even extremely-lowimpurity contents less than ppm from bulk and thin-film samples, in a nondestructive way. This is in contrast to other highly sensitive elemental-analysis methods such as inductively coupled plasma mass spectrometry, which can measure lower concentrations but is destructive and typically starts from a liquid phase (bulk samples), or secondary ion mass spectrometry, which is also destructive and can measure thin films directly. Applications for TES PIXE could include, for example, studies of low-level dopants or defects in semiconductors, with possibly the added bonus of chemical information. For example, a weak phosphorus line at $2.0 \mathrm{keV}$ is easily resolved even next to a very large Si $K$ peak at $1.84 \mathrm{keV}$ with TES PIXE. Mapping is also possible in the future by scanning the proton beam.

Another direction would be studies of biological specimens, as PIXE allows samples in air. For example, the demonstrated chemical sensitivity of Ti lines could be used to study the $\mathrm{Ti}$ oxidation state and the distribution of $\mathrm{Ti}$ in different organisms. This is especially interesting, as $\mathrm{Ti}$ compounds are used as anticancer drugs and because $\mathrm{Ti}$ is widely used as an implant material [45]. Naturally, other elements could be considered, as well.

\section{ACKNOWLEDGMENTS}

We thank S. Hornytzkyj for providing the pigment samples and M. Reis for discussions. This work is supported by the Finnish Funding Agency for Technology and Innovation TEKES, Academy of Finland Project No. 260880 and Academy of Finland Center of Excellence in Nuclear and Accelerator Based Physics (Ref. 251353), and by the NIST Innovations in Measurement Science program. M. R. J.P. thanks the National Graduate School in Materials Science for funding.

[1] Particle-Induced X-Ray Emission Spectrometry (PIXE), edited by S. A. E. Johansson, J. L. Campbell, and K. G. Malmqvist (Wiley, New York, 1995). 
[2] H. R. Verma, Atomic and Nuclear Analytical Methods (Springer, New York, 2007), Chap. 1.

[3] J. Szlachetko, M. Cotte, J. Morse, M. Salome, P. Jagodzinski, J.-Cl. Dousse, J. Hoszowska, Y. Kayser, and J. Susinia, Wavelength-dispersive spectrometer for x-ray microfluorescence analysis at the x-ray microscopy beamline ID21 (ESRF), J. Synchrotron Radiat. 17, 400 (2010).

[4] J. N. Ullom and D. A. Bennett, Review of superconducting transition-edge sensors for x-ray and gamma-ray spectroscopy, Supercond. Sci. Technol. 28, 084003 (2015).

[5] K. D. Irwin and G. C. Hilton, in Cryogenic Particle Detection, edited by Ch. Enss (Springer, New York, 2005).

[6] M. R. J. Palosaari, K. M. Kinnunen, J. Julin, M. Laitinen, M. Napari, T. Sajavaara, W. B. Doriese, J. Fowler, C. Reintsema, D. Swetz, D. Schmidt, J. Ullom, and I. J. Maasilta, Transition-edge sensors for particle induced x-ray emission measurements, J. Low Temp. Phys. 176, 285 (2014).

[7] J. Uhlig, W. Fullagar, J. N. Ullom, W. B. Doriese, J. W. Fowler, D. S. Swetz, N. Gador, S. E. Canton, K. Kinnunen, I. J. Maasilta, C. D. Reintsema, D. A. Bennett, L. R. Vale, G. C. Hilton, K. D. Irwin, D. R. Schmidt, and V. Sundström, Tabletop Ultrafast X-Ray Microcalorimeter Spectrometry for Molecular Structure, Phys. Rev. Lett. 110, 138302 (2013).

[8] J. N. Ullom, W. B. Doriese, D. A. Fischer, J. W. Fowler, G. C. Hilton, C. Jaye, C. D. Reintsema, D. S. Swetz, and D. R. Schmidt, Transition-edge sensor microcalorimeters for X-ray beamline science, Synchrotron Radiat. News, 27, 24 (2014).

[9] J. M. Gildemeister, A. T. Lee, and P. L. Richards, A fully lithographed voltage-biased superconducting spiderweb bolometer, Appl. Phys. Lett. 74, 868 (1999).

[10] D. A. Bennett, R. D. Horansky, D. R. Schmidt, A. S. Hoover, R. Winkler, B. K. Alpert, J. A. Beall, W. B. Doriese, J. W. Fowler, C. P. Fitzgerald, G. C. Hilton, K. D. Irwin, V. Kotsubo, J. A. B. Mates, G. C. O’Neil, M. W. Rabin, C. D. Reintsema, F. J. Schima, D. S. Swetz, L. R. Vale, and J. N. Ullom, A high resolution gamma-ray spectrometer based on superconducting microcalorimeters, Rev. Sci. Instrum. 83, 093113 (2012).

[11] K. D. Irwin, Appl. Phys. Lett. 66, 1998 (1995).

[12] J. A. Chervenak, E. N. Grossman, K. D. Irwin, John M. Martinis, C. D. Reintsema, C. A. Allen, D. I. Bergman, S. H. Moseley, and R. Shafer, Performance of multiplexed SQUID readout for Cryogenic Sensor Arrays, Nucl. Instrum. Methods Phys. Res., Sect. A 444, 107 (2000).

[13] W. B. Doriese, J. A. Beall, S. Deiker, W. D. Duncan, L. Ferreira, G. C. Hilton, K. D. Irwin, C. D. Reintsema, J. N. Ullom, L. R. Vale, and Y. Xu, Time-division multiplexing of high-resolution $\mathrm{x}$-ray microcalorimeters: Four pixels and beyond, Appl. Phys. Lett. 85, 4762 (2004).

[14] S. R. Bandler, R. P. Brekosky, A.-D. Brown, J. A. Chervenak, E. Figueroa-Feliciano, F. M. Finkbeiner, N. Iyomoto, R. L. Kelley, C. A. Kilbourne, F. S. Porter, J. Sadleir, and S. J. Smith, Performance of TES X-ray microcalorimeters with a novel absorber design, J. Low Temp. Phys., 151, 400 (2008).

[15] J. N. Ullom, J. A. Beall, W. B. Doriese, W. D. Duncan, L. Ferreira, G. C. Hilton, K. D. Irwin, C. D. Reintsema, and L. R. Vale, Optimized transition-edge X-ray microcalorimeter with $2.4 \mathrm{eV}$ energy resolution at $5.9 \mathrm{keV}$, Appl. Phys. Lett. 87, 194103 (2005).

[16] D. A. Wollman, K. D. Irwin, G. C. Hilton, L. L. Dulcie, D. E. Newbury, and J. M. Martinis, High-resolution, energydispersive microcalorimeter spectrometer for x-ray microanalysis, J. Microsc. 188, 196 (1997).

[17] For a review, see D. E. Newbury, K. D. Irwin, G. C. Hilton, D. A. Wollman, J. A. Small, and J. M. Martinis, in Cryogenic Particle Detection, edited by Ch. Enss (Springer, New York, 2005).

[18] Star Cryoelectronics LLC, Santa Fe, New Mexico, USA.

[19] M. A. Reis, P. C. Chaves, and A. Taborda, Radiative auger emission satellites observed by microcalorimeter-based energy-dispersive high-resolution PIXE, X-Ray Spectrom. 40, 141 (2011).

[20] G. C. Hilton, John M. Martinis, K. D. Irwin, N. E Bergren, D. A. Wollman, M. E. Huber, S. Deiker, and S. W. Nam, Microfabricated transition-edge $\mathrm{x}$-ray detectors, IEEE Trans. Appl. Supercond. 11, 739 (2001).

[21] J. N. Ullom, W. B. Doriese, G. C. Hilton, J. A. Beall, S. Deiker, W. D. Duncan, L. Ferreira, K. D. Irwin, C. D. Reintsema, and L. R. Vale, Characterization and reduction of unexplained noise in superconducting transition-edge sensors, Appl. Phys. Lett. 84, 4206 (2004).

[22] High Precision Devices Inc., Boulder, Colorado, USA.

[23] J. W. Fowler, B. K. Alpert, W. B. Doriese, Y.-I. Joe, G. C. O'Neil, J. N. Ullom, and D. S. Swetz, J. Low Temp. Phys. 184, 374 (2016).

[24] J. L. Campbell, N. I. Boyd, N. Grassi, P. Bonnick, and J. A. Maxwell, The Guelph PIXE software package IV, Nucl. Instrum. Methods Phys. Res., Sect. B 268, 3356 (2010).

[25] H. Paul and J. Sacher, Fitted empirical reference cross sections for K-shell ionization by protons, At. Data Nucl. Data Tables 42, 105 (1989).

[26] I. Orlic, C. H. Sow, and S. M. Tang, Experimental L-shell $\mathrm{X}$-ray production and ionization cross sections for proton impact, At. Data Nucl. Data Tables 56, 159 (1994).

[27] N. J. G. Pearce, W. T. Perkins, J. A. Westgate, M. P. Gorton, S. E. Jackson, C. R. Neal, and S. P. Cheney, A compilation of new and published major and trace element data for NIST SRM 610 and NIST SRM 612 glass reference materials, Geostandards Newsletter 21, 115 (1997).

[28] J. H. Hubbell and S. M. Seltzer, http://physics.nist.gov/ xaamdi.

[29] N. Kallithrakas-Kontos, X-ray chemical shift determination by energy dispersive detection, Spectrochim. Acta B Atom. Spectros. 51, 1655 (1996).

[30] J. Hasegawa, T. Tada, Y. Oguri, M. Hayashi, T. Toriyama, T. Kawabata, and K. Masai, Development of high-efficiency high-resolution particle-induced x-ray emission system for chemical state analysis of environmental samples, Rev. Sci. Instrum. 78, 073105 (2007).

[31] S. Contarini, P. A. W. van der Heide, A. M. Prakash, and Larry Kevan, Titanium coordination in microporous and mesoporous oxide materials by monochromated x-ray photoelectron spectroscopy and x-ray Auger electron spectroscopy, J. Electron Spectrosc. Relat. Phenom. 125, 25 (2002).

[32] S. Fukushima, T. Kimura, K. Nishida, V. A. Mihai, H. Yoshikawa, M. Kimura, T. Fujii, H. Oohashi, Y. Ito, and 
M. Yamashita, The valence state analysis of $\mathrm{Ti}$ in $\mathrm{FeTiO}_{3}$ by soft X-ray spectroscopy, Microchim. Acta 155, 141 (2006).

[33] K. F. J. Heinrich, C. E. Fiori, and R. L. Myklebust, Relative transition probabilities for the X-ray lines from the K level, J. Appl. Phys. 50, 5589 (1979).

[34] L. Rebohle, U. Lehnert, and G. Zschornack, $K \beta / K \alpha$ intensity ratios and chemical effects of some $3 \mathrm{~d}$ elements, X-Ray Spectrom. 25, 295 (1996).

[35] O. Sogut, E. Büyükkasap, and H. Erdogan, Chemical-effect variation of $K \beta K \alpha$ x-ray intensity ratios in $3 \mathrm{~d}$ elements, Radiat. Phys. Chem. 64, 343 (2002).

[36] G. Brunner, M. Nagel, E. Hartmann, and E. Arndt, Chemical sensitivity of the $K \beta / K \alpha$ x-ray intensity ratio for $3 \mathrm{~d}$ elements, J. Phys. B 15, 4517 (1982).

[37] J. Kawai, Chemical effects in the satellites of X-ray emission spectra, Nucl. Instrum. Methods Phys. Res., Sect. B 75, 3 (1993).

[38] R. L. Kauffman, J. H. McGuire, P. Richard, and C. F. Moore, Projectile and target dependence of the $K \alpha$ satellite structure, Phys. Rev. A 8, 1233 (1973).
[39] Y. Awaya, T. Kambara, and Y. Kanai, Multiple $K$ - and $L$-shell ionizations of target atoms by collisions with high-energy heavy ions, Int. J. Mass Spectrom. 192, 49 (1999).

[40] R. L. Watson, T. Chiao, and F. E. Jenson, Chemical Effect on $K \alpha$ X-Ray Satellites: New Evidence for Interatomic Transitions, Phys. Rev. Lett. 35, 254 (1975).

[41] O. Benka, R. L. Watson, and R. A. Kenefick, Resonant Electron Transfer in Ionic Fluorine Compounds following $1 s 2 p$ Ionization, Phys. Rev. Lett. 47, 1202 (1981).

[42] G. Demortier, Review of the recent applications of high energy microprobes in arts and archaeology, Nucl. Instrum. Methods Phys. Res., Sect. B 54, 334 (1991).

[43] T. O. Ziebold, Precision and sensitivity in electron microprobe analysis, Anal. Chem. 39, 858 (1967).

[44] P. J. Statham, in X-ray Spectrometry in Electron Beam Instruments, edited by D. B. Williams, J. I. Goldstein, and D. E. Newbury (Plenum, New York, 1995).

[45] K. M. Buettner and A. M. Valentine, Bioinorganic chemistry of titanium, Chem. Rev. 112, 1863 (2012). 